\title{
Leaky Integrate and Fire models coupled through copulas: association properties of the Interspike Intervals.
}

Sacerdote, Laura; Tamborrino, Massimiliano

Published in:

Chinese Journal of Physiology

Publication date:

2010

\section{Document version}

Publisher's PDF, also known as Version of record

Citation for published version (APA):

Sacerdote, L., \& Tamborrino, M. (2010). Leaky Integrate and Fire models coupled through copulas: association properties of the Interspike Intervals. Chinese Journal of Physiology, 53(6), 396-406.

http://www.cps.org.tw/docs/Vol53\%20No6P\%20Article\%206.pdf 


\title{
Leaky Integrate and Fire Models Coupled through Copulas: Association Properties of the Interspike Intervals
}

\author{
Laura Sacerdote ${ }^{1}$ and Massimiliano Tamborrino ${ }^{2}$ \\ ${ }^{1}$ Department of Mathematics, University of Torino Torino, Italy \\ and \\ ${ }^{2}$ Department of Mathematical Sciences, University of Copenhagen, Copenhagen, Denmark
}

\begin{abstract}
We propose a model able to describe the Interspike Intervals of two or more neurons subject to common inputs from the network. The single neuron dynamic is described through a classical Leaky Integrate and Fire model, but the model also catches the joint behavior of two neurons resorting to the use of copulas. Copulas are mathematical objects largely used to describe dependencies laws. Synchronous and delayed dependencies are considered by means of a set of examples. Results are discussed making use of crosscorrelograms.
\end{abstract}

Key Words: leaky integrate and fire models, copulas, interspike interval dependency

\section{Introduction}

Stochastic models have been extensively used to describe single neuron activity and to understand coding principles. Strong mathematical efforts have determined the development of analytical, numerical or eventually simulative methods for their study. This fact has allowed the use of single neuron models to describe the role of the noise in signal transmission and to study input-output relationships. In this framework Leaky Integrate and Fire (LIF) models owe their popularity to the existence of specific mathematical methods and to their ability to reproduce a set of experimental features $(4,5,9)$.

LIF models are of little help when one wishes to work with information transmitted in a neuronal network. The spike trains in a network are determined by connections between the involved neurons and groups of neurons. Available mathematical methods for the LIF models do not allow the description of Interspike Intervals (ISIs) in the presence of dependent processes. Jump diffusion processes have been proposed to model the dynamics of interconnected neurons (17) and other approaches make use of simplified LIF models to mimic the dynamic of single neuronal units linked with assigned laws (7). Simulation is the typical method for these studies, but it requires increasing computational time when the dimension of the network grows and the description of the single neuron dynamics is not oversimplifed. The lack of analytical results and the presence of a large number of parameters make the interpretation of simulations diffcult.

In this paper we propose a new approach to the description of the activity of neuronal networks. We present a model for the description of the simultaneous activity of two neurons influenced by dependent noise. The model is formulated as a LIF model. The reason for this is to get a mathematically tractable model, and to have the possibility to re-use results and methods developed for the single neuron case. The study is performed using simulations but this should only be considered as a first step devoted to the understanding of model features. Indeed, the

Corresponding author: Dr. Massimiliano Tamborrino, Department of Mathematical Sciences, University of Copenhagen, Universitetsparken 5, DK-2100 Copenhagen, Denmark. Phone: +45 35320736, E-mail: mt@ math.ku.dk, URL: http://www.math.ku.dk/ mt/

Received: November 13, 2009; Revised: January 27, 2010; Accepted: March 2, 2010.

(c)2010 by The Chinese Physiological Society and Airiti Press Inc. ISSN : 0304-4920. http://www.cps.org.tw 
structure of the model is such that further mathematical studies should allow the development of specific techniques for its analysis. We limit ourselves to model a couple of neurons but the extension to a larger number of units could be considered when the mathematical problems involved with the study of this model are solved.

The leading idea in the formulation of the model is the use of copulas. Recalling that a copula is a mathematical object joining two distributions to model the dependence between the involved random variables (r.v.), we introduce a dependence between two stochastic processes by coupling their increments. We describe the membrane potential evolution of each neuron by means of a diffusion process, and we couple the processes through suitable copulas joining their noise terms. The rationale for this type of coupling lies in the observation that both neurons are connected with the network. Hence, they become dependent due to their common dependence on the network activity. Other types of dependencies could be investigated, however, the main aim of this paper is to focus on this new approach and not to propose a new general model. Furthermore, we do not detail here a possible procedure to get a realistic dependence between the spike trains. We simply draft the basic ideas, postponing the mathematical procedure to a more technical paper to get a bivariate diffusion process as a limit of the Stein's model for two neurons. Hence, we do not build "ad hoc" families of copulas, but we use some of the most popular families to investigate the effect of the introduction of dependencies between spike trains modeled via LIF models.

A short review on copulas and their properties is presented in Section 2, while we refer to (12) for a complete introduction. The use of copula in neuroscience was first proposed by (8), but it is not yet sufficiently investigated. Here we describe each neuron via a LIF model, but the two involved stochastic processes are dependent to account for the dependency between the two neurons. In Section 3 we present the model and the quantities of interest for its study. The effect of using different copulas to couple the two stochastic processes is investigated in Section 4 to detect possible differences in the spike trains generated through different couplings of the modeled neurons and to recognize experimental features that the model can mimic. Hence, we illustrate the neuronal dynamics that can be explained through the proposed dependencies. In particular, we show that the model allows both synchronous and delayed spiking activity. A set of examples are discussed making use of crosscorrelograms.

Crosscorrelograms are the typical statistical method to analyze simultaneously recorded ISIs but some criticism on their use has appeared in the literature (2). The examples discussed here confirm these difficulties. Our results show that they are sensitive to different coupling intensities but they seem unable to distinguish between different types of dependencies. This fact is related to the global nature of the crosscorrelogram that considers the entire spike trains dependencies, but loses local ones. Statistical techniques to describe the local dependencies between two point processes are lacking. Further mathematical and statistical developments should be considered for a more complete study of the features of the proposed model. The copula approach is used here only to model the coupling of the noise terms in the membrane potential expressions. However, it will be applied to the study of dependencies in the simulated spike trains when the necessary mathematics will be developed.

The difficulty in the use of copulas between point processes suggests preliminary work on the coupling between random variables. A first step in this direction should be the determination of the copula between the first passage times through a boundary of the two coupled diffusion processes modeling the membrane potential evolution. This task implies the introduction of a renewal version of the proposed model and the discussion of a further set of examples. The limited space available for this paper discourages the presentation of these results here and we postpone this discussion to a future paper.

\section{Mathematical Background on Copulas}

In this Section we only consider bivariate random variables to avoid a heavy notation, but extensions to the multivariate case follow the same arguments. The typical object used to describe the statistical properties of a random variable $X$ is its distribution $F_{X}(x)=$ $\mathbb{P}(X<x), \forall x \in \mathbb{R}$. To describe a pair of random variables $(X, Y)$ one should know their marginal distributions $F_{X}(x), F_{Y}(y)$ and their joint distribution

$$
\mathbb{P}(X<x, Y<y)=F_{X, Y}(x, y), \forall x \in \mathbb{R}, y \in R .
$$

The marginal distributions can easily be obtained from the joint distribution by setting $x=\infty$ to get $F_{Y}(y)$ or $y=\infty$ to get $F_{X}(x)$. Hence, the bivariate distribution $F_{X, Y}(x, y)$ completely describes the pair of random variables catching both the marginal behavior and their dependencies. This fact has an immediate contraindication when one wishes to study dependencies. Indeed, the joint distribution merges dependencies and marginal features. To avoid this problem the notion of copula has been introduced and its use has gained popularity thanks to the increasing 

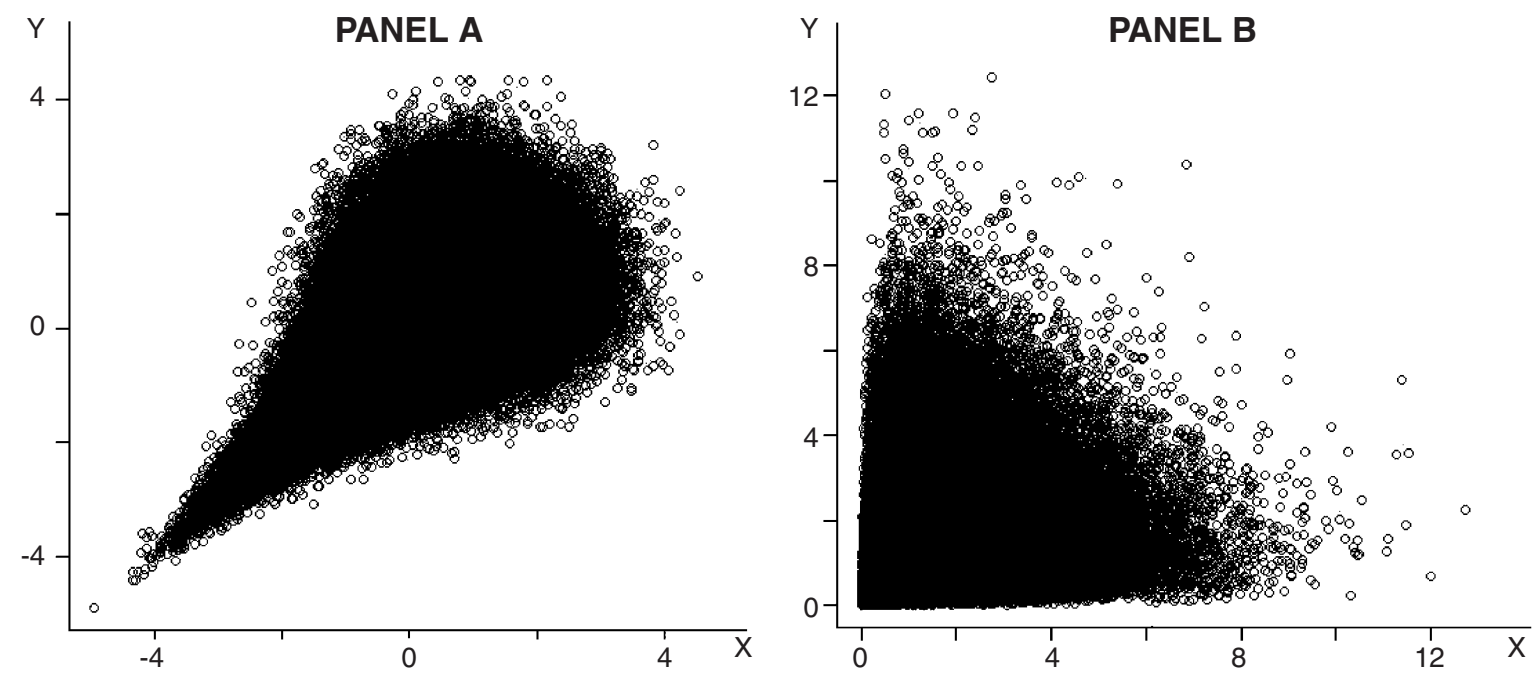

Fig. 1. Scatterplots of bivariate r.v.s $(X, Y)$ coupled through Clayton copulas with $\alpha=2$. Panel A: standard normal marginals. Panel B: exponential marginals with rate $r=1$.

number of modeling instances where it can be used. A huge number of papers makes use of copulas to model financial problems while applications in neuroscience up to now are rare $(8,16)$.

To define a copula between two r.v. let us consider two uniform r.v.s $U, V$ on $[0,1] \times[0,1]$. Assume that they are not necessarily independent, but are related through their joint distribution function

$$
C(u, v)=\mathbb{P}(U<u, V<v) \text {. }
$$

The function $C:[0,1] \times[0,1] \rightarrow[0,1]$ is called copula. Consider the two marginal distributions $F_{X}(x)=\mathbb{P}(X<x)$ and $F_{Y}(y)=\mathbb{P}(Y<y)$ of the two r.v.s $X$ and $Y$. It is easy to check that

$$
C\left(F_{X}(x), F_{Y}(y)\right)=F_{X, Y}(x, y)
$$

defines a bivariate distribution with marginals $F_{X}(x)$ and $F_{Y}(y)$. A celebrated theorem by Sklar establishes that any bivariate distribution can be written in the form of eq. [3]. Furthermore, if the marginals are continuous then the copula representation is unique (12).

If we change the marginals in [3] we obtain a different joint distribution. Furthermore, different marginals can be coupled through different copulas to obtain different bivariate distributions.

Copulas are scale free since they are invariant under increasing and continuous transformations. This property allows us to avoid assumptions on the scales of the marginals. Furthermore, the copulas contain all the information related to the dependencies between the random variables and do not involve marginal distributions. Hence, one can separate the study of bivariate distributions into two parts: the marginal behavior and the dependencies between the r.v.s contained in the copula structure.

The simplest bivariate copula is the independent copula which is defined as $C(u, v)=u v,(u, v) \in$ $[0,1] \times[0,1]$. More complex copula functions usually contain one or more parameters, which are called association parameters. If only one parameter appears in a copula function, it usually ref1ects the strength of the dependence. Consider for example the following copula function

$$
C(u, v)=\max \left\{\left(u^{-\alpha}+v^{-\alpha}\right)^{-1 / \alpha}, 0\right\}
$$

where $(u, v) \in[0,1] \times[0,1]$ and $\alpha \in(-1,0) \cup(0, \infty)$. It is known as a Clayton copula. In the limit when $\alpha \rightarrow 0$ it converges to the independence copula. A continuous copula $C(u, v)$ can be characterized through its probability density function, given by:

$$
c(u, v)=\frac{\partial^{2} C(u, v)}{\partial u \partial v} .
$$

For example, the independent copula has probability density function $c(u, v)=1,(u, v) \in[0,1] \times$ $[0,1]$, i.e. the dependence is uniform on $[0,1] \times[0,1]$. In Fig. 1 we show scatterplots of bivariate r.v.s coupled through the Clayton copula characterized by different marginals.

Many families of copulas have been proposed in the literature introducing copulas with different shapes that can solve a variety of modeling challenges. The most well-known families of copulas belong to the Archimedean family. These copulas owe their popularity to their large f1exibility. Furthermore, they are simple since their expression involves one single parameter. Unfortunately, they are based on 
algebraic arguments and their use can rarely be justified through modeling motivations. Research in this direction is still scarce and should be increased to give more significance to the use of copulas.

Specific indices able to measure the strength of dependencies between the involved r.v.s are often used in the study of bivariate models. Besides the correlation coefficient, a largely used association index is the so-called Kendall's $\tau$. It compares concordance and discordance probabilities for the involved r.v.s (12). It is defined as:

$$
\begin{aligned}
\tau= & \mathbb{P}\left[\left(X-X^{\prime}\right)\left(Y-Y^{\prime}\right)>0\right] \\
& -\mathbb{P}\left[\left(X-X^{\prime}\right)\left(Y-Y^{\prime}\right)<0\right]
\end{aligned}
$$

where $(X, Y)$ and $\left(X^{\prime}, Y^{\prime}\right)$ are two bivariate r.v.s identically distributed. Its statistical version is the estimator:

$$
\hat{\tau}=\frac{n_{c}-n_{d}}{\frac{1}{2} n(n-1)},
$$

where $n_{c}$ is the number of concordant pairs, $n_{d}$ is the number of discordant pairs in the data and $n$ is the sample size of each variable. Often it is advantageous to re-parametrize copulas through Kendall's $\tau$ in substitution of the original parameter. In the case of a Clayton copula the relationship between $\alpha$ and Kendall's $\tau$ is $\alpha=\frac{2 \tau}{1-\tau}$.

\section{The Model}

We describe the subthreshold membrane potential evolution of two neurons through two Ornstein Uhlenbeck processes:

$$
\begin{aligned}
& d X_{t}=\left(-\frac{X_{t}}{\theta}+\mu_{1}\right) d t+\sigma_{1} d W_{1}(t) ; X_{t_{0}}=0 \\
& d Y_{t}=\left(-\frac{Y_{t}}{\theta}+\mu_{2}\right) d t+\sigma_{2} d W_{2}(t) ; X_{t_{0}}=0 .
\end{aligned}
$$

Here $\theta>0$ is the time decaying membrane constant, $\mu_{1}$ and $\mu_{2}$ describe the net input impinging on the neurons while $\sigma_{1}$ and $\sigma_{2}$ describe the intensity of its variability. According to the classical assumptions on LIF models, eqs. [8] and [9] describe the subthreshold behavior while the interspike intervals of the two neurons are described by the first passage time of the processes through a boundary $S$ :

$$
T_{1}=\min \left(t>t_{0}: X_{t}>S ; X_{t_{0}}<S\right)
$$

$$
T_{2}=\min \left(t>t_{0}: Y_{t}>S ; Y_{t_{0}}<S\right) .
$$

After the spike each membrane potential is instantaneously reset to its resting value that we assume equal to zero. When the two neurons are not coupled, $W_{1}(t)$ and $W_{2}(t)$ are two independent Wiener processes. For a fixed $n>0$ let us consider a partition of $\left(t_{0}, t\right)$ with steps $h=\frac{t-t_{0}}{n}$. The Euler discretized version of these equations is

$$
\begin{aligned}
& X_{i}=X_{i-1}+\left(-\frac{X_{i-1}}{\theta}+\mu_{1}\right) h+\sigma_{1} Z_{i}^{1} ; X_{t_{0}}=0 \\
& Y_{i}=Y_{i-1}+\left(-\frac{Y_{n-1}}{\theta}+\mu_{2}\right) h+\sigma_{2} Z_{i}^{2} ; \quad Y_{t_{0}}=0 .
\end{aligned}
$$

Here $Z_{i}^{k}=W_{k}(i h)-W_{k}((i-1) h)$ are independent Gaussian random variables with mean 0 and variance $h$ for $k=1,2$ and $i=1,2, \ldots, n$. In the case of independent neurons the r.v.s $\left\{Z_{i}^{1}\right\}$ and $\left\{Z_{i}^{2}\right\}$ are independent. However, when the two neurons belong to a network they are linked to each other. Hence, we cannot assume that their dynamics are independent. In particular, both neurons are subject to a variability depending on the whole network. Then it seems reasonable to introduce a dependence between the random variables $\left(Z_{i}^{1}, Z_{i}^{2}\right) \mathrm{i}=1,2, \ldots, n$, representing the increments of the membrane potential at times $t_{i}=$ $i h$. Due to the assumptions on LIF models these random variables have Gaussian marginal distributions. We introduce specific copula expressions to describe the coupling of the increments to couple the ISIs of the two considered neurons. Assume that their joint distribution is

$$
F_{Z_{i}^{1}}, Z_{i}^{2}\left(z_{1}, z_{2}\right)=\mathrm{C}\left(\phi\left(z_{1}\right), \phi\left(z_{2}\right)\right),
$$

where $\phi(\cdot)$ indicates the Gaussian distribution with mean 0 and variance $h$. This approach also allows the introduction of a delay in the coupling law. Indeed, instead of eq. (13) we can assume:

$$
F_{Z_{i}^{1}, Z_{i+m}^{2}}\left(z_{1}, z_{2}\right)=C\left(\phi\left(z_{1}\right), \phi\left(z_{2}\right)\right), i=1,2, \ldots, n .
$$

Here $m$ introduces a delay of $m h$ in the coupling between the increments. Eq. [14] allows us to model connections between neurons that are not instantaneous but require a time interval to be effective.

We further assume that after eliciting of spike, the membrane potential of each neuron restarts from its resting value and its increments maintain the coupling with the other neuron. Note that this hypothesis implies a renewal assumption for the single 
neuron behavior. However, the dependence between the increments of the two processes destroys the renewal property for the joint dynamics.

With a slight abuse of terminology, we will call OU model the discretized model [11], [12] with the dependency law [13], while we call it "delayed OU model" when the copula is given by [14].

Having intracellular recordings of the membrane potential evolutions, the choice of the copula functions [13] or [14] could be suggested by statistical arguments. Alternatively, one could resort to model motivations to get a specific expression for the selected copula function. For example, one could resort to the Stein's model

$$
\begin{aligned}
d V_{i}(t) & =\left(-\frac{V_{i}(t)}{\theta}+\mu_{i}\right) d t+\delta^{+} d N_{i}^{+}(t)+\delta^{-} d N_{i}^{-}(t) \\
i & =1,2
\end{aligned}
$$

where $\theta$ and $m_{i}, i=1,2$ have the same meaning as in eqs. [11], [12], $\delta^{+}$and $\delta^{-}$are the amplitudes of instantaneous changes of the membrane potential values due to the arrival of external inputs to the neuron, and $N_{i}^{+}(t)$ and $N_{i}^{-}(t)$ are two independent Poisson processes. When the two neurons belong to independent subnetworks, one can assume that the processes $\left(N_{1}^{+}(t), N_{1}^{-}(t)\right)$ are independent from $N_{2}^{+}(\mathrm{t})$, $\left.N_{2}^{-}(t)\right)$. However, when the neurons belong to an interconnected network these processes are no more independent due to the presence of some common inputs. These last inputs may eventually have a delayed effect on one of the two neurons but in any case they determine a dependence between the two neurons. Different coupling laws may arise according to the rules introduced to account for common inputs. This determines the copulas catching the different coupling between the epochs of excitatory (inhibitory) inputs. When the continuous limit of Stein's equations [15] is performed one gets [8] and [9] $(10,15)$ and the increments of the processes exhibit a dependence due to the dependence between the arrival times of the inputs. The copula expression between the increments should then be determined from the copula between the arrival times. This task requests some mathematical effort and we postpone it to a future paper. Here we simply focus on the consequences of the coupling of the two equations through well known families of copulas. Future statistical and mathematical studies will allow the selection of realistic families of copulas for the proposed model.

To facilitate our understanding on some consequences of the hypothesized coupling of the processes [11] and [12], we consider the special case of the perfect integrator model, taking the limit of [11] and [12] as $\theta$ goes to $\infty$ when the coupling is determined by eq. [13]. The r.v.s corresponding to the state of the two processes at time $\mathrm{n}$ can be rewritten as

$$
\begin{aligned}
& X_{n}=X_{n-1}+\mu_{1} h+\sigma_{1} Z_{n}^{1}=n \mu_{1} h+\sigma_{1} \sum_{i=1}^{n} Z_{i}^{1} ; X_{t_{0}}=0 \\
& Y_{n}=Y_{n-1}+\mu_{2} h+\sigma_{2} Z_{n}^{2}=n \mu_{2} h+\sigma_{2} \sum_{i=1}^{n} Z_{i}^{2} ; Y_{t_{0}}=0
\end{aligned}
$$

Repeating the afore-mentioned abuse of terminology we will refer to the discretized model [16], [17] with the dependency law [13] as the Wiener model.

Recalling the invariance of the copula function for monotone transformations, the copula between $\left(X_{n}, Y_{n}\right)$ coincides with the copula between $\left(\sum_{i=1}^{n} Z_{i}^{1}\right.$, $\sum_{i=1}^{n} Z_{i}^{2}$ ). Indeed $X_{n}$ and $Y_{n}$ are strictly increasing functions of $\sum_{i=1}^{n} Z_{i}^{1}$ and $\sum_{i=1}^{n} Z_{i}^{2}$, respectively (12).

Hence the couple $\left(X_{n}, Y_{n}\right)$, when $n \rightarrow \infty$, tends to a bivariate normal vector, due to the multivariate central limit theorem (1) applied to the sum of random variables involved in [16] and [17] and the copula coupling such r.v.s becomes the normal copula (12). Analogous discussion can be repeated in the case of coupling through eq. [14]. The case of the OU discretized model [11] and [12] is less straightforward, but an analogous convergence result holds. In the next section, we will study the effect of the use of different copulas between increments on the ISI's properties. To this aim, it is useful to separate instances in which the number $n$ of iterations at the time of boundary crossing is large enough to determine the Gaussian convergence of the copula joining $\left(X_{n}, Y_{n}\right)$, from those in which the copula between the processes is significantly different from the Gaussian one. In we report the results obtained for the Clayton copula between $\left(X_{n}, Y_{n}\right)$ for some simulated examples. The numbers marked with bold character indicate where the convergence to the normal copula for the coupled r.v.s has not yet happened. Here we test the convergence to the normal copula through a Goodness-offit test for copulas (6) with $p$-value $p=0.05$. In the next Section we select the examples of ISIs determined by a non Gaussian copula that we compare with those determined by a Gaussian copula, i.e. the copula associated with the bivariate normal distribution (12). We have repeated this analysis also for the Frank and the Gumbel copulas. These copulas are Archimedean ones (12). The Frank copula is defined by

$$
C(u, v)=-\frac{1}{\alpha} \ln \left(1+\frac{\left(e^{-\alpha u}-1\right)\left(e^{-\alpha v}-1\right)}{e^{-\alpha}-1}\right)
$$


Table 1.

\begin{tabular}{|c|c|c|c|c|c|c|c|}
\hline \multirow[t]{2}{*}{$\tau$} & \multirow[t]{2}{*}{$n_{\text {clayton }}$} & \multicolumn{2}{|c|}{$\mu=2$} & \multicolumn{2}{|c|}{$\mu=1.5$} & \multicolumn{2}{|c|}{$\mu=0.4$} \\
\hline & & $n_{T_{1}}$ & $n_{T_{2}}$ & $n_{T_{1}}$ & $n_{T_{2}}$ & $n_{T_{1}}$ & $n_{T_{2}}$ \\
\hline 0.5 & 25 & 63 & 78 & 78 & 96 & 333 & 360 \\
\hline 0.8 & 200 & 53 & 50 & 81 & 67 & 885 & 325 \\
\hline 0.96 & 100 & 52 & 52 & 80 & 80 & 1051 & 1047 \\
\hline-0.5 & 100 & 48 & 63 & 55 & 78 & 514 & 250 \\
\hline-0.96 & 400 & 48 & 64 & 57 & 65 & 514 & 242 \\
\hline
\end{tabular}

Experimental evaluation on the convergence of the Clayton copula between $\left(X_{n}, Y_{n}\right)$ (given by eq. 16, 17, Wiener model) to the normal one is made as follows. Let $n_{\mathrm{T}_{1}}\left(n_{\mathrm{T}_{2}}\right)$ be the number of iterations of the Euler schema until the boundary crossing for neuron 1 [2], for different values of $\mu$ and $\tau$. Let $n_{\text {Clayton }}$ be the number of terms necessary for convergence to the normal copula to occur. If $\max \left(n_{\mathrm{T}_{1}}, n_{\mathrm{T}_{2}}\right)<n_{\text {Clayton }}$ then we mark $\left(n_{\mathrm{T}_{1}}, n_{\mathrm{T}_{2}}\right)$ with bold character.

where $(u, v) \in[0,1] \times[0,1]$ and $\alpha \neq 0$, while the Gumbel copula is defined by

$$
C(u, v)=\exp \left(-\left[(-\ln u)^{\alpha}+(-\ln v)^{\alpha}\right]^{1 / \alpha}\right.
$$

where $(u, v) \in[0,1] \times[0,1]$ and $\alpha \in[1, \infty)$. For both these copulas we obtained similar values to those in the second column of Table 1, but these last two families tend to anticipate the convergence to the normal copula with respect to the Clayton one.

\section{Results}

Here we discuss the results making use of crosscorrelograms. Hence, our analysis cannot be complete since crosscorrelograms capture global properties of spike trains, and do not capture local dependencies. To get a more complete analysis of the model features, one should develop suitable alternative mathematical methods. Unfortunately, this is not yet the case and further studies appear to be necessary. A possible approach could make use of the notion of copulas to measure dependencies between interspike times. To motivate these efforts it seems reasonable to start with a simulation study collecting expected features of the model and analyzing them with crosscorrelograms. Indeed, a first analysis of the model properties can suggest its improvement or eventually discourage its future use.

The model has been studied to ascertain the following features:

1. the differences between couples of spike trains described by means of OU or Wiener processes;

2. the role of the choice of the copula family on the coupled behavior of the two modeled neurons;

3. the role of different intensities of Kendall's $\tau$ between the increments of the processes on the dependence features of the simulated spike trains;
4. the consequences of the introduction of a delay in the coupling of the increments of the processes on the dependency properties of the simulated spike trains.

To perform our analysis we have simulated spike trains from models [11], [12]. In all the considered examples, we have fixed $\sigma_{1}^{2}=\sigma_{2}^{2}=1 \mathrm{mV}^{2} / \mathrm{ms}$, $S=10 \mathrm{mV}$ and $\theta=20 \mathrm{~ms}$. The case $\theta=\infty$, corresponding to the Wiener model, is also considered. Different values for the input excesses $\mu_{1}$ and $\mu_{2}$ are considered to distinguish the subthreshold $\left(S>\mu_{i} \theta, i=1,2\right)$ and suprathreshold regimes $\left(S<\mu_{i} \theta, i=1,2\right)$. Furthermore, we have considered different copulas to join the increments of the processes [11], [12]. Copulas of Frank, Gumbel and Clayton have been used for different values of the Kendall's $\tau: \tau=-0.96,-0.5,0.5$, $0.8,0.96$.

As far as the first point is concerned, we have compared crosscorrelograms obtained using eqs [11] and [12] with those obtained using [16] and [17]. In both models we coupled the increments through [13]. Indeed, we wanted to understand if the same coupling law applied to different marginal dynamics, i.e. the OU or the Wiener models, may give rise to different features. We have collected a set of examples for different ranges of $\tau$ and for different choices of copulas. In Fig. 2 panels A-C (B-D) we show the crosscorrelograms between ISIs simulated from the Wiener (OU) model, using the Clayton copula for $\mu=1,2 \mathrm{mVms}^{-1}$ and $\tau=-0.5,0.96$. Here and in the next crosscorrelograms, the dotted line represents the reference level in crosscorrelation, i.e. the predicted expected level for stationary independent processes $(3,14)$. The full lines represent the 0.95 confidence interval for the reference level. Thus a flat crosscorrelogram indicates two independent processes. Fig. 2 shows that the qualitative features of the two models are similar. Indeed, when $\tau$ is negative both models 



Fig. 2. Panel A(B): crosscorrelograms (crosscorrelation denoted by $\xi$ ) between ISIs simulated via eqs. [16]-[17], Wiener model and (11)-(12), OU model with $\mu=1,2 \mathrm{mV} / \mathrm{ms}$ and coupled through Clayton Copula with $\tau=-0.5$. Upper panels $\mu=1 \mathrm{mV} / \mathrm{ms}$, lower panels $\mu=2 \mathrm{mV} / \mathrm{ms}$. Panel C(D): the same structure of Panel A(B) with $\tau=0.96$. Other parameters: $\sigma_{1}=\sigma_{2}=1$ $\mathrm{mV}^{2} / \mathrm{ms}^{-1}, S=10 \mathrm{mV}, \theta=20 \mathrm{~ms}, t_{0}=0 \mathrm{~ms}, x_{0}=0 \mathrm{mV}$.

give rise to a strong simultaneous inhibition while positive $\tau$ gives rise to synchronism in the spike times when $\mu$ is large enough. Note that the crosscorrelograms of both models present bumps around zero, i.e. the model determines an inhibitory effect in cor- respondence to simultaneous spikes, and simultaneity is a frequent phenomenon. To understand the causes of this result, we have considered the autocorrelograms for the ISIs of each simulated neuron, in a set of instances characterized by large $\tau$ and a suprath- 

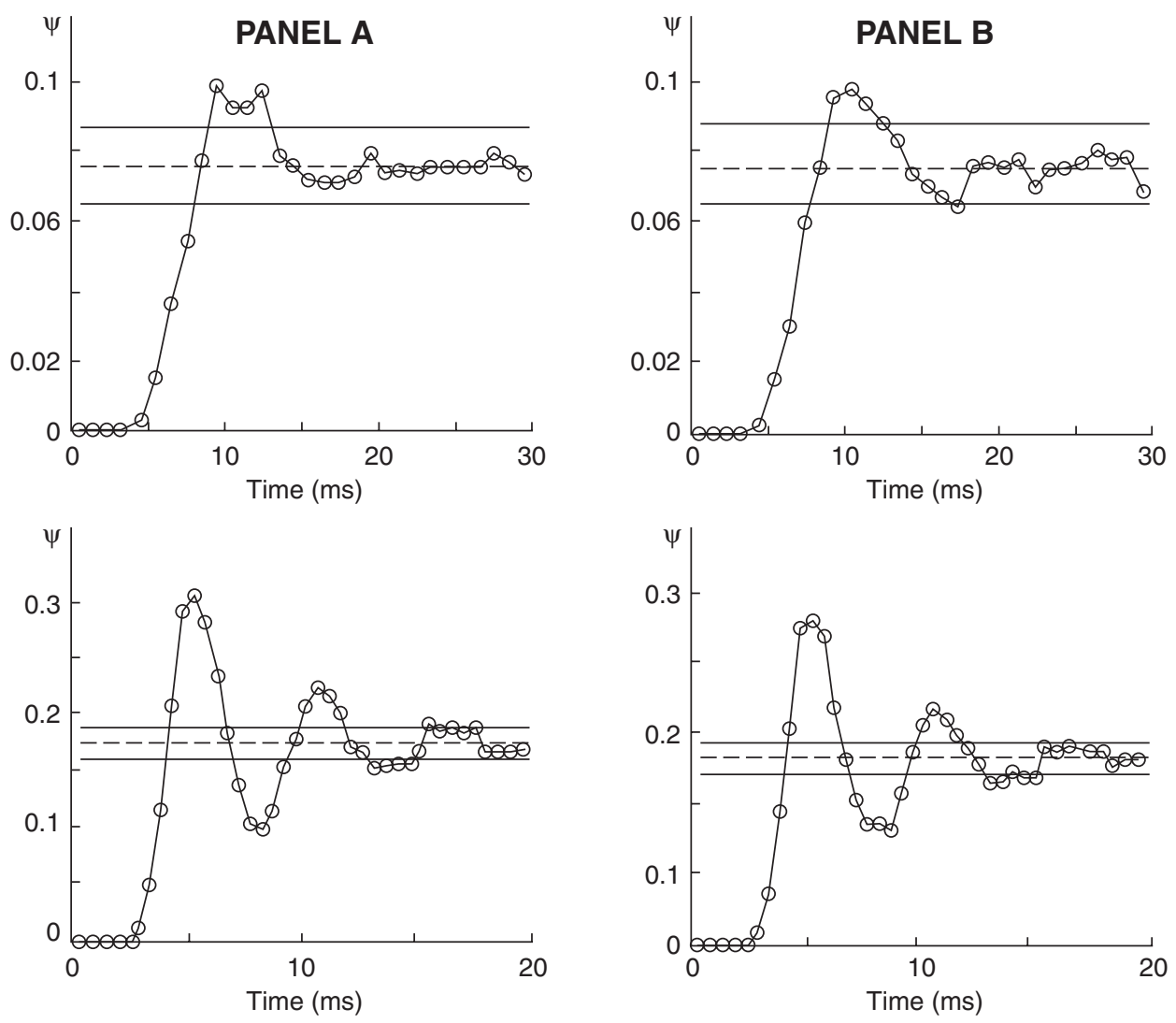

Fig. 3. Panel A(B): autocorrelograms (autocorrelation denoted by $\psi$ ) of neuron 1 (2) for the OU model. Upper panels $\mu=1 \mathrm{mV} / \mathrm{ms}$, lower panels $\mu=2 \mathrm{mV} / \mathrm{ms}$. Other parameters: the same values assigned in Fig. 2. When the coupling copula is the Clayton one with $\tau=0.96$, the corresponding crosscorrelograms are illustrated in Fig. 2 Panel D.

reshold regime.

The examples in Fig. 3 show autocorrelograms corresponding to the crosscorrelograms of the $\mathrm{OU}$ model in Fig. 2. Here and in the next autocorrelograms, the dotted lines represent the reference level in autocorrelation, that is the predicted asymptotic value $1 / k$ where $k$ denotes the mean of the spike-interval data. The full lines represent the 0.95 confidence interval related to the reference level (13).

For the considered choice of parameters, i.e. for $\mu$ large enough, the autocorrelogram reveals a periodicity, related to the dominance of the deterministic behavior of the OU process when $\mu$ is large. This means that the observed bumps in the crosscorrelograms in Fig. 2 depend on the marginal behavior of each neuron. Hence, from the considered examples, we can conclude that the models react in similar way to the introduction of a dependency in their membrane potential evolution while their differences can be explained by their marginal behavior, being characterized by different first passage time distributions. As a consequence of these results, in the remaining part of this paper we limit ourselves to the study of the OU model.

In the second step of our analysis, we check a possible change of dependence in the spike trains when different copulas are used to couple the increments. To pursue this task, we select the study cases making use of Table 1, wishing to avoid false coupling similarities determined by the convergence to the normal copula of the couples $\left(X_{n}, Y_{n}\right)$ when $n$ is large enough. Hence, we consider only cases for which the threshold crossing of at least one process happens before the convergence to the normal copula. In Fig. 4 Panel A we show scatterplots of bivariate r.v.s coupled through Clayton and Gumbel copulas used to couple the Gaussian increments when $\tau=0.8$ while in Panel B we show the corresponding crosscorrelograms for the OU model when $\mu=2 \mathrm{mV} / \mathrm{ms}$. No significant differences can be observed in Fig. 4 corresponding to a change of the used copula.

Fig. 5 illustrates the effect of changes in the strength of the coupling, measured through the Kendall's $\tau$ in the subthreshold regime. The crosscorrelogram does not detect weak dependencies while strong positive dependencies determine synchronous activity and negative ones determine inhibition of such synchronism. A further remark concerns the case $\tau=0.96$. Indeed the bumps of the crosscorrelogram cannot be explained looking at the auto- 



Fig. 4. Panel A: scatterplots of bivariate r.v.s $(X, Y)$ coupled through Clayton (upper panel) and Gumbel (lower panel) copulas with $\tau=0.8$ and standard normal marginals. Panel B: corresponding crosscorrelograms for the OU model when $\mu=2 \mathrm{mV} / \mathrm{ms}$. Other parameters: the same values assigned in Fig. 2. The parameter choice for $\mu$ is such that the processes coupled with Gumbel copula behave like processes coupled with normal copula at the crossing time while in the case of the Clayton copula such convergence is not yet verified.

correlograms as we did with those in Fig. 2. Hence, the presence of a strong positive dependence determines an inhibition phenomenon around the synchronism not explained by the marginal behavior of each neuron. This case corresponds to different behaviors of the Wiener and OU models since the subthreshold regime does not exist for the Wiener model, and the crosscorrelograms of the Wiener model under analogous conditions do not exhibit bumps (data not shown).

Finally we have considered the effect of different delay values in the delayed OU model. The introduction of the delay determines small shifts of the peak around zero of the crosscorrelograms. The shifts are positive or negative according to the type of positive or negative dependency (data not shown). Only large values for the delay, i.e. values at least of the order of the mean firing time, make this shift visible. The limited effect on the crosscorrelograms may again be explained by the technique used for the model analysis. Indeed, the crosscorrelograms work on the entire spike train and thus they average over many values. This fact may hide some existing dependence.

We have also performed a preliminary study on the role of $\sigma$ values in the introduced coupling between the two neuronal dynamics. Qualitative features observed do not change, but high values of $\sigma$ destroy synchronization or mutual inhibition.

\section{Conclusions}

We have proposed a model for a pair of neurons belonging to a network. The dependence between different units is introduced, postulating the existence of a copula, joining the increments of the two processes. Further statistical and modeling studies should be performed to determine realistic expressions for this copula while here we considered classical families of copulas without motivating their choice with model properties.

The use of autocorrelograms and crosscorrelograms has allowed us to show that the model can reproduce a set of observed features such as synchronization and mutual inhibition between the two modeled neurons. A variant of the model allows us to reproduce delays in the synchronization or in the inhibition. As one may expect, increasing positive dependencies determine higher positive peaks in the crosscorrelograms, while negative dependencies determine depressions in their shapes. In the case of strong positive dependencies, one observes bumps in the 
PANEL A
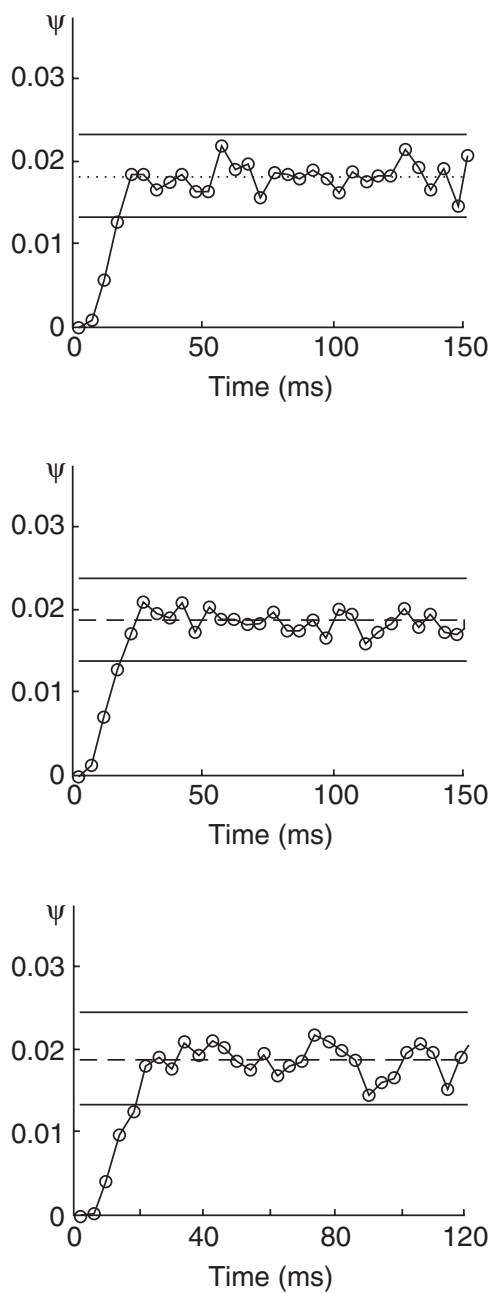

PANEL B
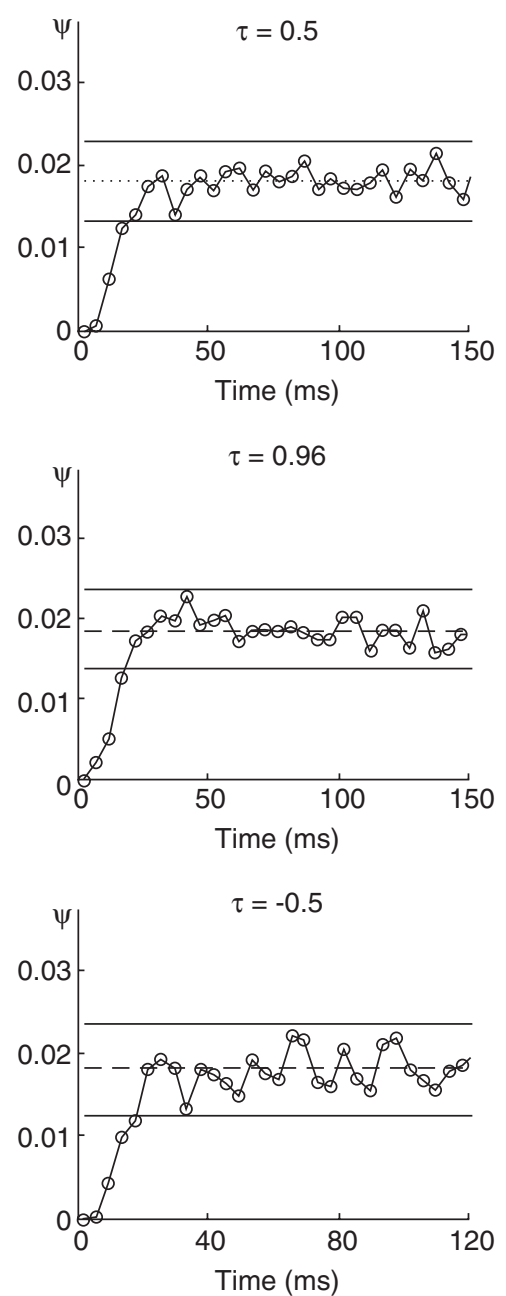

PANEL C
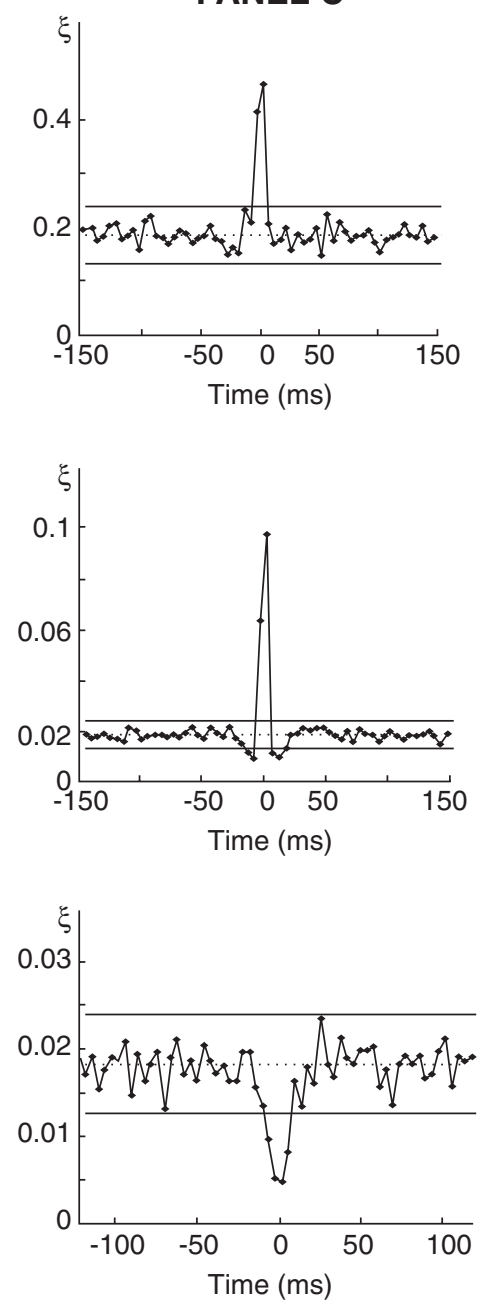

Fig. 5. Panel A(B): autocorrelograms of neuron 1(2). Panel C: crosscorrelograms for the OU model coupled with Clayton copula for $\tau=0.5,0.96,-0.5$ and $\mu=0.4 \mathrm{mV} / \mathrm{ms}$. Other parameters: the same values assigned in Fig. 2.

crosscorrelograms. In the suprathreshold regime these bumps can be explained by the autocorrelograms that exhibit peaks, but in the subthreshold regime this phenomenon has no analogous explanation. Indeed in this case it is related to the introduced dependency between the two neurons.

Different models and different types of dependencies have been analyzed, and it seems that these changes do not have important consequences.

However, our conclusion could be related to our investigation technique. Indeed, the crosscorrelogram does not detect local dependencies, and further investigations with different methods should check the possible existence of these. Hence, we cannot yet claim with security the equivalence of different coupling rules on the two spike trains.

We conclude by observing that many generalizations of this model can be done but to make it convenient to study larger networks, it is necessary to develop mathematical and statistical methods that avoid simulations, and to analyze the results with more specific methods than crosscorrelograms. This mathematical task could start with the study of simplified situations such as a renewal model in which both neurons restart after the spike of the second one. Despite its loss of realism, the study could be a first step to develop the necessary mathematical methods. This method could then hopefully be used for the more complex case.

\section{Acknowledgments}

This work has been performed under partial support by MIUR PRIN 2008.

\section{References}

1. Billingsey, P. Probability and Measure. New York: John Wiley \& Sons, 1995.

2. Borisyuk, G.N., Borisyuk, R.M., Kirillov, A.B., Kovalenko, E.I. and Kryukov, V.I. A new statistical method for identifying inter- 
connections between neuronal network elements. Biol. Cybern. 52: 301-306, 1985.

3. Brillinger, D.R. Condence intervals for the crosscovariance function. Selecta: Statistica Canadiana V: 1-16, 1979.

4. Burkitt, A.N. A review of the integrate-and-fire neuron model: I. Homogeneous synaptic input. Biol. Cybern. 95: 119, 2006.

5. Burkitt, A.N. A review of the integrate-and-fire neuron model: II. Inhomogeneous synaptic input and network properties. Biol. Cybern. 95: 97-112, 2006.

6. Genest, C., Rémillard, B. and Beaduoin, D. Goodness-of-fit tests for copulas: A review and a power study. Insurance: Math. Econ. 44: 199-213, 2009.

7. Iglesias, J. and Villa, A.E.P. Emergence of preferred firing sequences in large spiking neuronal networks during simulated neuronal development. Int. J. Neural. Syst. 18: 267-277, 2008.

8. Jenison, R.L. and Reale, R.A. The shape of neural dependence. Neural Comput. 16: 665-672, 2004.

9. Jolivet, R., Roth, A., Schurmann, F., Gerstner, W. and Senn, W. Special issue on quantitative neuron modeling. Biol. Cybern. 99: 237-239, 2009.

10. Kallianpur, G. On the diffusion approximation to a discontinuous model for a single neuron. In: Contributions to Statistics, edited by Sen, P.K. Amsterdam: North-Holland, pp. 247-258, 1983.

11. Kloeden, P. and Platen, P. The Numerical Solution of Stochastic Differential Equations. Berlin: Springer Verlag, 1992.

12. Nelsen, R. An Introduction to Copulas. New York, NY: Springer, 2006.

13. Perkel, D.H., Gerstein, G.L. and Moore, G.P. Neuronal spike trains and stochastic point processes. I. The single spike train. Biophys. J. 7: 391-418, 1967.

14. Perkel, D.H., Gerstein, G.L. and Moore, G.P. Neuronal spike trains and stochastic point processes. II. Simultaneous spike trains. Biophys. J. 7: 419-440, 1967.

15. Ricciardi, L.M. Diffusion Processes and Related Topics in Biology. Lecture Notes in Biomathematics. Berlin: Springer Verlag, vol. 14, 1977.

16. Sacerdote, L. and Sirovich, R. A copulas approach to neuronal networks models. J. Physiol. Paris, 103: 223-230, 2010.

17. Sacerdote, L., Sirovich, R. and Villa, A.E.P. Effect of increasing inhibitory inputs on information processing within a small network of spiking neurons. Lect. Notes Comput. Sci. 4507: 23-30, 2007. 